\section{DOI: $\underline{10.15863 / \mathrm{TAS}}$ \\ International Scientific Journal Theoretical \& Applied Science}

p-ISSN: 2308-4944 (print) e-ISSN: 2409-0085 (online)

Year: $2015 \quad$ Issue: $01 \quad$ Volume: 21

Published: $30.01 .2015 \quad$ http://www.T-Science.org
Denis Alexandrovich Chemezov Master of Engineering and Technology, Corresponding member of International Academy TAS, Lecturer of Vladimir Industrial College, Russia chemezov-da@yandex.ru

SECTION 7. Mechanics and machine construction.

\title{
COMPUTER DESIGN AND ANALYSIS OF PRESSURE DISTRIBUTION ON THE SURFACE OF THE BLADE OF THE IMPELLER RADIAL TURBINE
}

\author{
Abstract: The paper presents three-dimensional design of the impeller geometry of the blade radial turbine. The \\ pressures acting on the surface of the blade during operation of the turbine visually presented on the interactive plot. \\ Key words: blade, impeller of turbine, model. \\ Language: Russian \\ Citation: Chemezov DA (2015) COMPUTER DESIGN AND ANALYSIS OF PRESSURE DISTRIBUTION \\ ON THE SURFACE OF THE BLADE OF THE IMPELLER RADIAL TURBINE. ISJ Theoretical \& Applied \\ Science 01 (21): 1-6. DOI: http://dx.doi.org/10.15863/TAS.2015.01.21.1
}

\section{УДК 621.224}

\section{КОМПЬЮТЕРНОЕ КОНСТРУИРОВАНИЕ И АНАЛИЗ РАСПРЕДЕЛЕНИЯ ДАВЛЕНИЯ НА ПОВЕРХНОСТЬ ЛОПАСТИ РАБОЧЕГО КОЛЕСА РАДИАЛЬНОЙ ТУРБИНЫ}

Аннотация: В работе представлено трехмерное конструирование рабочего колеса по геометрическим параметрам лопасти радиальной турбины. Величины давлений действующих на поверхности лопасти в процессе эксплуатации турбины визуально представлены на интерактивной эпюре.

Ключевые слова: лопасть, рабочее колесо турбины, модель.

Гидравлическая турбина - лопаточная машина, приводится во вращение потоком жидкости для преобразования механической энергии потока жидкости в механическую энергию на валу [1]. В зависимости от напора и мощности гидравлической установки, выбирают тип гидравлической турбины.

Радиально-осевые турбины считаются быстроходными. В этих турбинах, поток жидкости движется в двух направлениях:

a) радиальном - от периферии к центру вращающегося рабочего колеса турбины;

б) осевом - течение жидкости к выходу.

Радиальная турбина состоит из ротора и статора. Ротор - рабочее колесо, состоящее из ступицы, соединенной с валом турбины, комплекта лопастей (детали, имеющие сложные криволинейные поверхности по всей длине) и обода [2].

Конструирование гидравлической турбины методом объемного твердотельного моделирования, позволяет получить точную геометрию машины и в дальнейшем выполнять инженерные расчеты отдельных деталей или сборочного узла в САЕ-системах.

Модель рабочего колеса радиальной турбины строилась в интегрированной среде Ansys Workbench. Генерация геометрии рабочего колеса турбины со всеми элементами, на первом этапе моделирования выполнялась в модуле BladeGen [3]. Были заданы следующие значения параметров: координаты ( $x \quad u z)$, по которым производится расчет размеров и конфигурации лопастей и обода рабочего колеса турбины; размеры входной и выходной кромок лопасти; толщина лопасти; количество лопастей. В меридианном сечении (рис. 1) указаны элементы рабочего колеса радиальной турбины [4]: $a-$ входная зона; $b-$ внешний обвод (ведомый); $c-$ втулочная поверхность (ведущий обвод); $d-$ выходная зона; $e, f$ - линии тока. В диалоговом окне программы будут представлены следующие характеристики моделируемого рабочего колеса: шаг (в градусах) входной и выходной кромки 
лопасти; центр инерции $Z, R, T, M_{p}, \quad M$; аэродинамическая поверхность (область) лопасти [5]; длина биссектрисы угла, образованного сторонами лопасти в поперечном сечении; длина выпуклости лопасти; меридианная длина лопасти; угол установки лопасти [6]; отношение длины хорды лопасти к шагу.
Все данные импортировались в модуль Transient Structural - DesignModeler [7], где были получены трехмерные модели лопасти (рис. 2) и рабочего колеса турбины (рис. 3) массой 8,217 кг, наружным диаметром 200 мм и шириной 95 мм

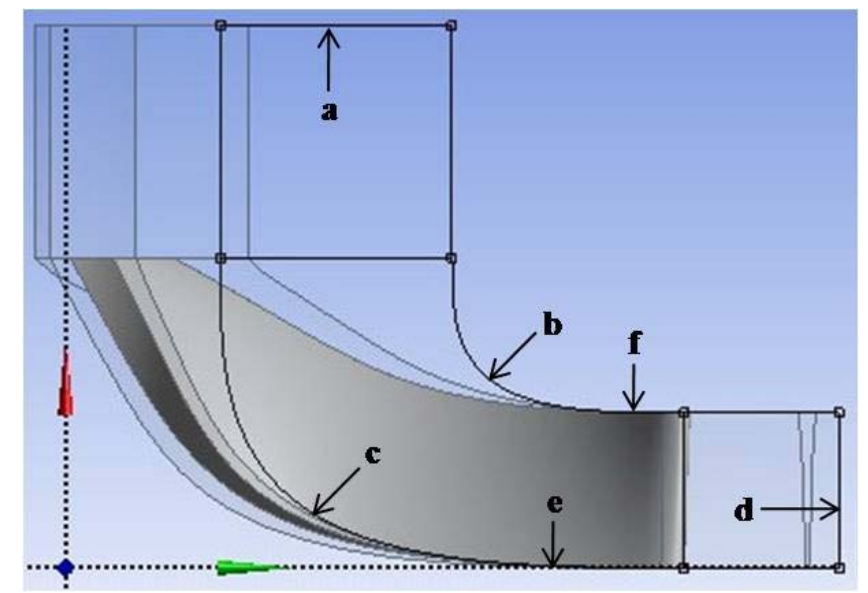

Рисунок 1 - Меридианное сечение рабочего колеса радиальной турбины.

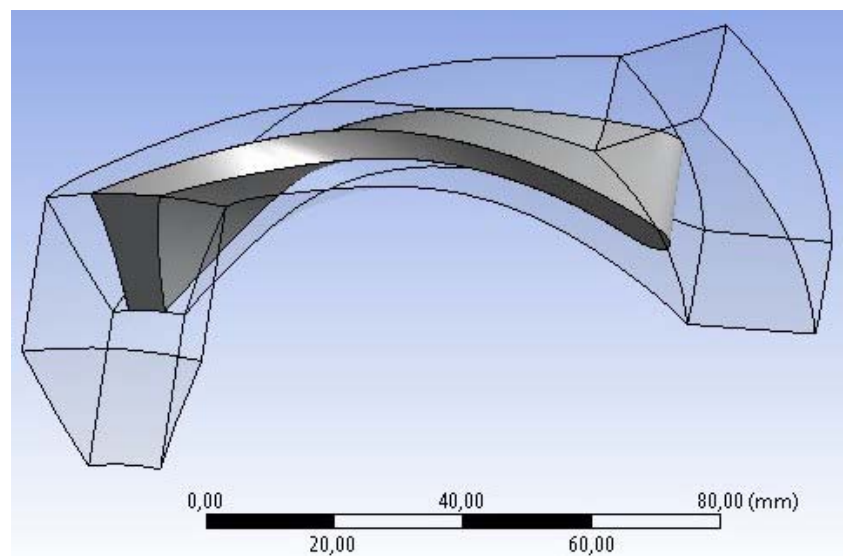

Рисунок 2 - Трехмерная модель лопасти рабочего колеса радиальной турбины.

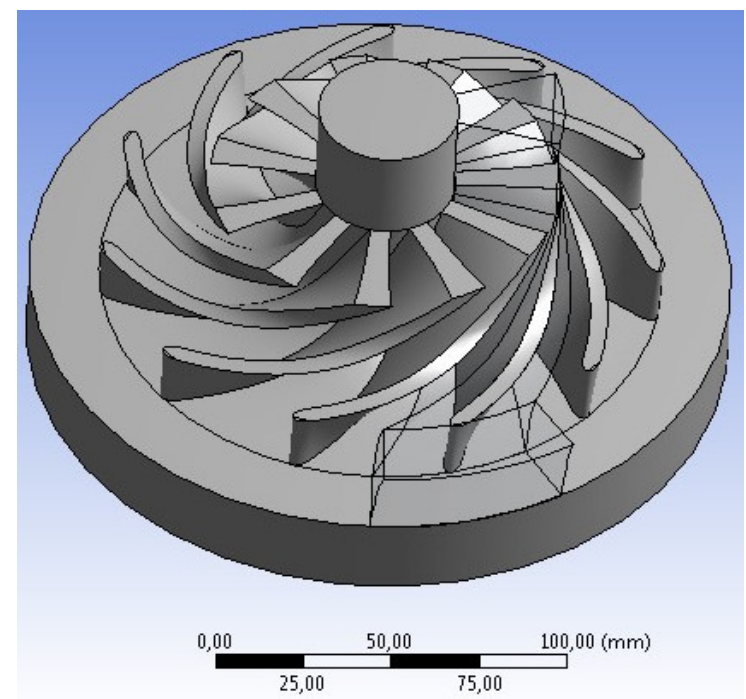

Рисунок 3 - Трехмерная модель рабочего колеса радиальной турбины. 
Широкие функциональные возможности программы BladeGen позволяют анализировать геометрические характеристики рабочего колеса турбины. Меридианный контур может быть представлен в виде цветной гаммы, нанесенной на эскиз сечения смоделированного рабочего колеса радиальной турбины. Цвета дают качественную оценку величин параметров: Tema (Theta) - угол охвата лопасти в плане (рис. 4, а); Бета (Beta) угол между хордой и плоскостью вращения лопасти (рис. 4, б) [8]; угол наклона лопасти (рис. 4, в) [9]; нормальная толщина лопасти (рис. 4, г).

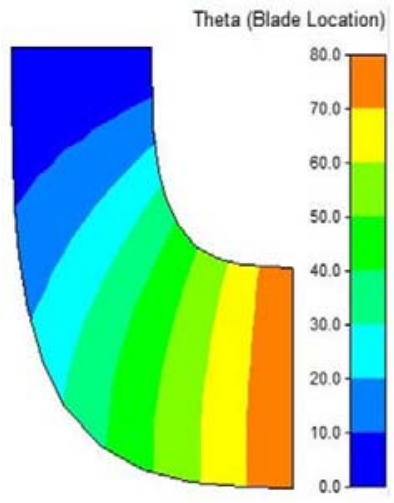

a)

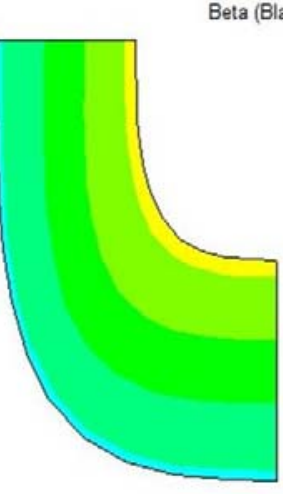

б)

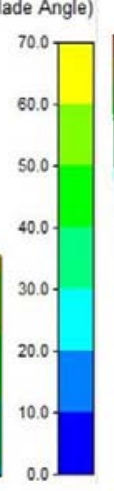

Рисунок 4 - Эпюры меридианных контуров.

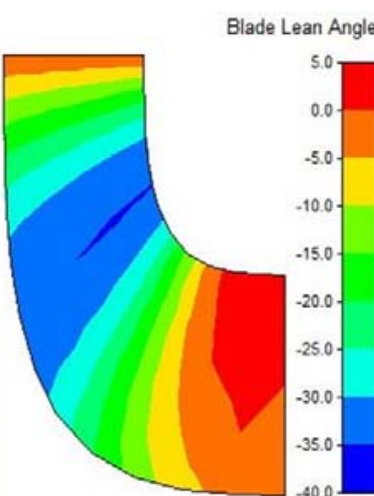

в)

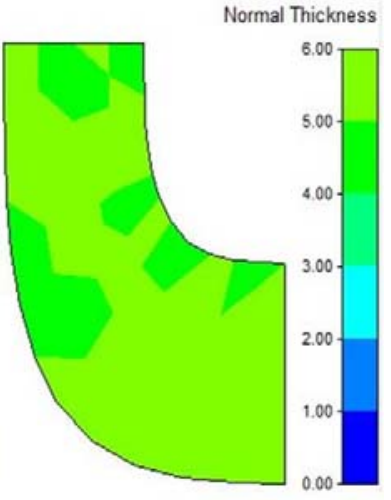

r)
Кривизна втулочной поверхности и внешнего обвода рабочего колеса (меридиан) радиальной турбины и кривизна лопасти рассчитывалась при обратном радиусе кривизны $0 \ldots 0,1$, входной кромке лопасти равной 0 и выходной кромке лопасти равной 1 . Зависимости представлены в табл. $1-2$.

Значения углов Tета и Бета входной и выходной кромок лопасти получены на промежутке от 0 до 1. Зависимости углов кромок лопасти рабочего колеса радиальной турбины представлены в табл. 3 - 4 .

Отношение входной кромки к выходной кромке лопасти рассчитано при втулочной поверхности рабочего колеса равной 0 и внешнем обводе равном 1. Зависимости отношений представлены в табл. 5 .
Величины угла наклона (меридиан 0 - 1) получены при постоянной и переменной длинах лопасти. Зависимости угла представлены в табл. 6.

В табл. 7. представлены значения площади (квазиортогональный параметр) рабочего колеса турбины, при входной кромке лопасти равной 0 и выходной кромке лопасти равной 1 .

Характеристика аэродинамической поверхности лопасти рабочего колеса турбины на всей длине отображена в табл. 8.

Результаты расчета максимального сферического диаметра между двумя соседними лопастями, при входной кромке лопасти равной 0 и выходной кромке лопасти равной 1 , представлены в табл. 9.

Таблица 1

Значения кривизны втулочной поверхности и внешнего обвода рабочего колеса (меридиан) радиальной турбины.

\begin{tabular}{|c|c|c|c|c|c|c|c|}
\hline \multicolumn{8}{|c|}{ Втулочная поверхность рабочего колеса } \\
\hline Входная - выходная кромки & $-0,2$ & 0,22 & 0,5 & 0,78 & 0,92 & 1,2 \\
\hline Обратный радиус кривизны & 0 & 0,005 & 0,027 & 0,023 & 0,003 & 0 \\
\hline Радиус, мм & 103,2 & 62,87 & 36,5 & 20,8 & 20,0 & 20,0 \\
\hline Расстояние вдоль меридианной кривой, мм & $-19,2$ & 21,12 & 48,01 & 74,9 & 88,34 & 115,23 \\
\hline Внешний обвод рабочего колеса & $-0,2$ & 0,22 & 0,5 & 0,78 & 0,92 & 1,2 \\
\hline Входная - выходная кромки & 0 & 0,008 & 0,058 & 0,032 & $1,57 \times 10^{-15}$ & 0 \\
\hline Обратный радиус кривизны & 94,6 & 72,33 & 57,79 & 50,16 & 50,0 & 50,0 \\
\hline Радиус, мм & $-10,6$ & 11,66 & 26,5 & 41,35 & 48,77 & 63,61 \\
\hline
\end{tabular}


Значения кривизны лопасти рабочего колеса радиальной турбины (средняя линия).

\begin{tabular}{|c|c|c|c|c|}
\hline Входная - выходная кромки & 0 & 0,3 & 0,6 & 1,0 \\
\hline Обратный радиус кривизны & $5,84 \times 10^{-5}$ & $2,8 \times 10^{-5}$ & $2,81 \times 10^{-6}$ & $-5,42 \times 10^{-6}$ \\
\hline Радиус, мм & 80 & 54,21 & 29,9 & 20,0 \\
\hline Расстояние вдоль меридианной кривой, мм & 0 & 25,8 & 51,61 & 86,02 \\
\hline Расстояние вдоль биссектрисы, мм & 0 & 29,34 & 58,69 & 97,81 \\
\hline
\end{tabular}

Таблица 3

Значения угла Тета входной и выходной кромок лопасти рабочего колеса радиальной турбины.

\begin{tabular}{|c|c|c|c|c|c|}
\hline \multicolumn{6}{|c|}{ Угол Тета входной кромки лопасти } \\
\hline Входная - выходная кромки & 0 & 0,25 & 0,5 & 0,75 & 1,0 \\
\hline Угол Тета, град. & 0 & 0 & 0 & 0 & 0 \\
\hline Радиус, мм & 80,0 & 80,0 & 80,0 & 80,0 & 80,0 \\
\hline Расстояние вдоль меридианной кривой, мм & 0 & 0 & 0 & 0 & 0 \\
\hline \multicolumn{6}{|c|}{ Угол Тета выходной кромки лопасти } \\
\hline Входная - выходная кромки & 0 & 0,25 & 0,5 & 0,75 & 1,0 \\
\hline Угол Тета, град. & 79,99 & 79,99 & 79,99 & 79,99 & 79,99 \\
\hline Радиус, мм & 20,0 & 27,5 & 35,0 & 42,5 & 50,0 \\
\hline Расстояние вдоль меридианной кривой, мм & 86,02 & 75,27 & 64,52 & 53,76 & 43,01 \\
\hline
\end{tabular}

Таблица 4

Значения угла Бета входной и выходной кромок лопасти рабочего колеса радиальной турбины.

\begin{tabular}{|c|c|c|c|c|c|}
\hline \multicolumn{6}{|c|}{ Угол Бета входной кромки лопасти } \\
\hline Входная - выходная кромки & 0 & 0,25 & 0,5 & 0,75 & 1,0 \\
\hline Угол Бета, град. & 28,424 & 36,529 & 45,129 & 53,942 & 62,57 \\
\hline Радиус, мм & 80,0 & 80,0 & 80,0 & 80,0 & 80,0 \\
\hline Расстояние вдоль меридианной кривой, мм & 0 & 0 & 0 & 0 & 0 \\
\hline \multicolumn{6}{|c|}{ Угол Бета выходной кромки лопасти } \\
\hline Входная - выходная кромки & 0 & 0,25 & 0,5 & 0,75 & 1,0 \\
\hline Угол Бета, град. & 28,427 & 36,471 & 45,087 & 53,923 & 62,571 \\
\hline Радиус, мм & 20,0 & 27,5 & 35,0 & 42,5 & 50,0 \\
\hline Расстояние вдоль меридианной кривой, мм & 86,02 & 75,27 & 64,52 & 53,76 & 43,01 \\
\hline
\end{tabular}

Таблица 5

Отношение входной кромки к выходной кромке лопасти рабочего колеса радиальной турбины.

\begin{tabular}{|c|c|c|c|c|c|}
\hline \multicolumn{7}{|c|}{ Входная кромка лопасти - эллипс с малым радиусом } \\
\hline Втулочная поверхность - внешний обвод & 0 & 0,25 & 0,5 & 0,75 & 1,0 \\
\hline Значение параметров & 2,5 & 2,5 & 2,5 & 2,5 & 2,5 \\
\hline Входная кромка лопасти - эллипс с большим радиусом & 0,25 & 0,5 & 0,75 & 1,0 \\
\hline Втулочная поверхность - внешний обвод & 0 & 5,5 & 5,0 & 5,0 & 5,0 \\
\hline Значение параметров & 5,0 & 5,0 & 5,0 \\
\hline
\end{tabular}

Таблица 6

Значения угла наклона лопасти рабочего колеса радиальной турбины.

\begin{tabular}{|c|c|c|c|c|c|c|}
\hline \multicolumn{7}{|c|}{ Постоянная длина лопасти } \\
\hline Втулочная поверхность - внешний обвод & 0 & 0,2 & 0,4 & 0,6 & 0,8 & 1,0 \\
\hline Угол наклона, град. & 0 & 15,26 & 19,02 & 15,45 & 8,56 & $6,52 \times 10^{-7}$ \\
\hline Радиус, мм & 80,0 & 67,09 & 54,27 & 42,42 & 35,89 & 35,0 \\
\hline Расстояние вдоль меридианной кривой, мм & 0 & 12,9 & 25,8 & 38,71 & 51,61 & 64,51 \\
\hline \multicolumn{7}{|c|}{ Переменная длина лопасти } \\
\hline Втулочная поверхность - внешний обвод & 0 & 0,2 & 0,4 & 0,6 & 0,8 & 1,0 \\
\hline Угол наклона, град. & 0,31 & 36,21 & 44,91 & 34,7 & 4,53 & $-0,45$ \\
\hline Радиус, мм & 80,0 & 67,33 & 55,51 & 44,96 & 35,82 & 35,0 \\
\hline Расстояние вдоль меридианной кривой, мм & $-0,002$ & 12,67 & 24,62 & 36,01 & 52,06 & 64,55 \\
\hline
\end{tabular}


Таблица 7

Значения площади (квазиортогональный параметр) рабочего колеса радиальной турбины.

\begin{tabular}{|c|c|c|c|c|c|c|}
\hline \multicolumn{8}{|c|}{ Без учета лопастей } \\
\hline Входная - выходная кромки & 0 & 0,2 & 0,4 & 0,6 & 0,8 & 1,0 \\
\hline Площадь, мм ${ }^{2}$ & 10053,1 & 9133,65 & 8723,99 & 7890,46 & 6907,28 & 6597,34 \\
\hline \multicolumn{7}{|c|}{ С учетом лопастей } \\
\hline Входная - выходная кромки & 0 & 0,2 & 0,4 & 0,6 & 0,8 & 1,0 \\
\hline Площадь, мм & \\
\hline
\end{tabular}
Значения площади аэродинамической поверхности лопасти рабочего колеса радиальной турбины.

\begin{tabular}{|c|c|c|c|c|c|c|}
\hline Входная - выходная кромки & 0 & 0,2 & 0,4 & 0,6 & 0,8 & 1,0 \\
\hline Площадь, мм ${ }^{2}$ & 483,52 & 469,21 & 459,73 & 455,26 & 455,86 & 461,47 \\
\hline
\end{tabular}

Таблица 9 Значение максимального сферического диаметра между двумя соседними лопастями рабочего колеса радиальной турбины.

\begin{tabular}{|c|c|c|c|c|c|c|}
\hline Входная - выходная кромки & 0 & 0,201 & 0,398 & 0,594 & 0,798 & 1,0 \\
\hline Максимальный сферический диаметр, мм & 20,0 & 20,25 & 22,13 & 27,21 & 30,54 & 30,0 \\
\hline
\end{tabular}

На всей длине лопасти рабочего колеса будет действовать переменное давление, создаваемое движущейся в радиальном направлении жидкостью (для расчета принята вода). Для расчета величин давления приняты: плотность воды $\left(1000\right.$ кг/ $\left.\mathrm{M}^{3}\right)$, температура воды $\left(22^{\circ} \mathrm{C}\right)$ и векторная величина - гидростатическое ускорение $\left(3,5 \mathrm{M} / \mathrm{c}^{2}\right)$. Численные значения результатов моделирования представлены цветовой гаммой на поверхности трехмерной модели лопасти (рис. 5).

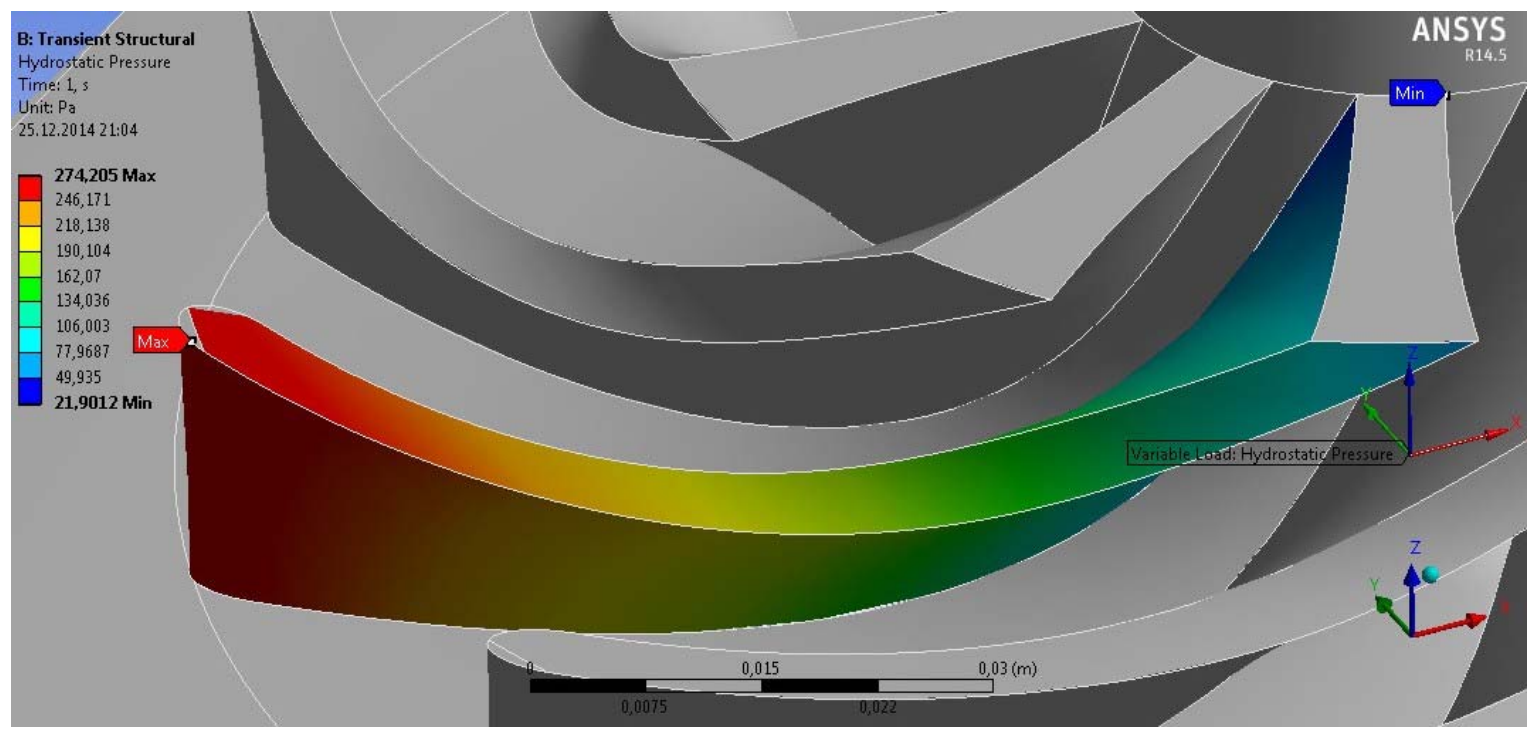

Рисунок 5 - Распределение давления (Па) на поверхности лопасти рабочего колеса радиальной турбины.

Отмечено, что наибольшее давление возникает на входной кромке лопасти рабочего колеса, а наименьшее - на выходной. При этом величина давления изменяется более чем в десять pa3.
Интегрированный модуль BladeGen в среде Ansys - это построение объемных твердотельных моделей и детальный анализ геометрии элементов турбин различных конфигураций на этапе проектирования реальной лопастной машины. 
Комплексные инженерные расчеты и анализ результатов выполняются путем разбиения объемной модели рабочего колеса на заданное количество узлов/элементов (Mesh) c последующей передачей данных в модули Autodyn, CFX, Finite Element Modeler, Fluent, ICEM CFD, Mechanical APDL и Polyflow.

\section{References:}

1. (2015)

Turbina.

Available: https://ru.wikipedia.org/wiki/\%D2\%F3\%F0\%E 1\%E8\%ED\% $\%$ E0 (Accessed: 02.01.2015).

2. (2015) Radialno-osevaya turbina. Available: https://ru.wikipedia.org/wiki/ $\% \mathrm{D} 0 \% \mathrm{E} 0 \% \mathrm{E} 4 \% \mathrm{E}$ $8 \% \mathrm{E} 0 \% \mathrm{~EB} \% \mathrm{FC} \% \mathrm{ED} \% \mathrm{EE}-$ $\% \mathrm{EE} \% \mathrm{~F} 1 \% \mathrm{E} 5 \% \mathrm{E} 2 \% \mathrm{E} 0 \% \mathrm{FF} \quad \% \mathrm{~F} 2 \% \mathrm{~F} 3 \% \mathrm{~F} 0 \% \mathrm{E}$ 1\%E8\%ED\%E0 (Accessed: 02.01.2015).

3. (2015) ANSYS BladeModeler. Available: http://www.cae-expert.ru/product/ansysblademodeler (Accessed: 02.01.2015).

4. (2015) Postroenie geometrii protochnoy chasti V Design Modeler. Available: http://studentengineer.pro/index.php?page=ansys/cfx/cfxgeo mDM (Accessed: 02.01.2015).

5. (2015) Aerodinamicheskaya poverhnost. Available:

http://www.2zn.ru/aerodinamicheskaya poverh nost.html (Accessed: 02.01.2015).
6. (2015) Ugly ustanovki lopasti. Available: http://www.aviationsweb.ru/page-448.html (Accessed: 02.01.2015).

7. (2015) Transient Structural Analysis. Available: https://www.sharcnet.ca/Software/Fluent14/hel $\mathrm{p} / \mathrm{wb} \operatorname{sim} / \mathrm{ds}$ transient mechanical analysis ty pe.html (Accessed: 02.01.2015).

8. (2015) Ugly theta I beta dlya opisaniya geometrii lopatki turbomashiny. Available: http://www.cae-club.ru/content/ugly-theta-ibeta-dlya-opisaniya-geometrii-lopatkiturbomashin (Accessed: 02.01.2015).

9. (2015) Ugly - naklon - lopast. Available: http://www.ngpedia.ru/id532580p1.html (Accessed: 02.01.2015). 